\title{
CÓMO AFRONTAR LA PANDEMIA DEL CORONAVIRUS (COVID-19) EN LAS AMÉRICAS: RECOMENDACIONES Y LÍNEAS DE ACCIÓN SOBRE SALUD MENTAL
}

Miguel Gallegos ${ }^{\text {a, b \& c1 }}{ }^{\mathbb{D}}$, Carlos Zalaquett ${ }^{\mathrm{d}}{ }^{\mathbb{D}}$, Sandra Elizabeth Luna

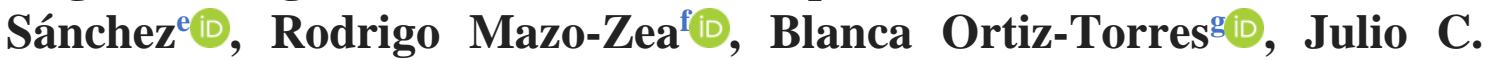
Penagos-Corzo $^{\mathrm{h}}\left(\mathbb{D}\right.$, Nelson Portillo ${ }^{\mathrm{i}}{ }^{(0}$, Ivelisse Torres Fernández $^{\mathrm{j}}(\mathbb{D}$, Alfonso Urzúa ${ }^{\mathrm{E}}$, Melissa Morgan Consoli ${ }^{1}$, Fernando Andrés

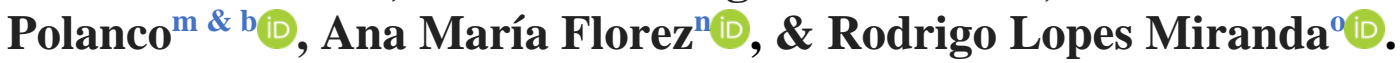

Universidad Católica del Maule, Talca, Chilea ; Consejo Nacional de Investigaciones Científicas y Técnicas, Buenos Aires, Argentina ${ }^{\mathrm{b}}$; Universidad Nacional de Rosario, Rosario, Argentina ; The Pennsylvania State University, State College, United States of Americad; Universidad Francisco Marroquín, Ciudad de Guatemala, Guatemala ${ }^{\mathrm{e}}$ : Universidad Pontificia Bolivariana, Medellín, Colombiaf; Universidad de Puerto Rico, San Juan, Puerto Rico ; Universidad de las Américas Puebla, San Andrés Cholula, México ; Boston College, Chestnut Hill, United States of America; Universidad Carlos Albizu, San Juan, Puerto Rico; ${ }^{\mathrm{j}}$ Universidad Católica del Norte, Antofagasta, Chile ${ }^{\mathrm{k}}$; University of California, Santa Barbara, United States of America ; Universidad Nacional de San Luis, San Luis, Argentina $^{\mathrm{m}}$; Asociación Panameña de Psicólogos, Panamá, Panamán; Universidade Católica Dom Bosco, Campo Grande, Brasil ${ }^{\circ}$.

\section{RESUMEN}

El 11 de marzo del 2020 la Organización Mundial de la Salud declaró pandemia a la enfermedad ocasionada por el Covid-19, la cual provoca efectos tanto en la salud física como mental de las personas infectadas por el virus, así como en el personal sanitario que las asiste. Dada la escasa disponibilidad de guías y recomendaciones elaboradas por instituciones psicológicas a nivel continental para afrontar los efectos en la salud mental, investigadores de la Sociedad Interamericana de Psicología (SIP) se propusieron el objetivo de construir una guía de recomendaciones sobre diferentes temas y problemáticas psicológicas. Para esto, se realizó una revisión sistemática de la literatura científica referida a la afectación de la salud mental provocada por este tipo de pandemia. Con base en la información recuperada y analizada de diversas bases de datos científicos (PsycInfo, Scielo, Redalyc y Dialnet), fuentes documentales procedentes de organismos nacionales e internacionales de salud mental, consulta a expertos en salud mental y diversas investigaciones científicas, se redactó un documento orientador para afrontar las consecuencias emocionales y psicosociales del Covid-19. Se espera que este aporte sea de utilidad para orientar a la ciudadanía y las prácticas profesionales de los psicólogos y psicólogas, y se constituya en una herramienta que sustente la toma de decisiones en las organizaciones psicológicas y en los organismos gubernamentales en salud pública de los diferentes países de las Américas.

Palabras Claves

COVID-19; intervenciones psicológicas; salud mental; guía; Sociedad Interamericana de Psicología

${ }^{1}$ Correspondence about this article should be addressed to Miguel Gallegos: $\underline{\text { maypsi@yahoo.com.ar }}$ 


\begin{abstract}
On March 11, 2020, the World Health Organization declared a pandemic caused by Covid-19, a disease that affects both the physical and mental health of people infected with the virus, as well the health personnel who assist them. Given the limited availability of guides and recommendations prepared by psychological institutions at a continental level to face the effects on mental health, researchers from the Inter-American Society of Psychology (SIP) have created some recommendations on different psychological issues and problems relate to the disease and pandemic. To accomplish this this, a systematic review of the scientific literature on mental health issues caused by this type of pandemic was carried out. Based on the information recovered and analyzed from various scientific databases (PsycInfo, Scielo, Redalyc and Dialnet), documentary sources from national and international mental health organizations, consultation with experts in mental health and various scientific investigations, we have created the following guiding document for facing the emotional and psychosocial consequences of Covid-19. It is hoped that this contribution will be useful in guiding both laypeople and professional psychologists, and will become a tool to support decision-making in psychological organizations and public health government agencies of different countries of the Americas.
\end{abstract}

\title{
Keywords
}

COVID-19; psychological interventions; mental health; guide; Interamerican Society of Psychology

\section{RESUMO}

Em 11 de março de 2020, a Organização Mundial da Saúde declarou uma pandemia para a doença causada pelo Covid-19, que gerou efeitos na saúde física e mental das pessoas infectadas pelo vírus, bem como nos profissionais de saúde que assistem os infectados. Dada a disponibilidade limitada de guias e recomendações preparadas por instituições psicológicas em nível continental para enfrentar os efeitos na saúde mental, pesquisadores da Sociedade Interamericana de Psicologia (SIP) estabeleceram o objetivo de elaborar um guia de recomendações sobre diferentes questões e problemas psicológicos. Para tanto, foi realizada uma revisão sistemática da literatura científica referente aos impactos sobre a saúde mental causada por esse tipo de pandemia. Assim, apresenta-se um documento de orientação para enfrentar as consequências emocionais e psicossociais do Covid-19 com base em informações recuperadas e analisadas em várias bases de dados científicos (PsycInfo, Scielo, Redalyc e Dialnet), fontes documentais de organizações nacionais e internacionais de saúde mental, consulta com especialistas em saúde mental e várias investigações científicas. Espera-se que essa contribuição seja útil para orientar as pessoas e as práticas profissionais de psicólogas e psicólogos, e se torne uma ferramenta para apoiar a tomada de decisões em organizações psicológicas e em agências governamentais de saúde pública de diferentes países das Américas.

\section{Palavras-chave}

COVID-19; intervenções psicológicas; saúde mental; guia; Sociedade Interamericana de Psicologia 


\section{COPING WITH THE CORONAVIRUS (COVID-19) PANDEMIC IN THE AMERICAS: RECOMMENDATIONS AND GUIDELINES FOR MENTAL HEALTH \\ LIDANDO COM A PANDEMIA DE CORONAVÍRUS (COVID-19) NAS AMÉRICAS: RECOMENDAÇÕES E LINHAS DE AÇÃO EM SAÚDE MENTAL}

\section{Introducción}

Desde la emergencia de la pandemia, los diferentes gobiernos de las América y el mundo han implementado diversas medidas de contención del Covid-19. Muchas de ellas, como la cuarentena2, el aislamiento3 o distanciamiento físico4, no sólo implicaron una discontinuidad de las pautas habituales de interacción social y el cambio en los hábitos de comportamiento individual y colectivo, sino también diversas consecuencias a nivel psicosocial y de la salud mental en la toda la población (American Psychological Association [APA], 2020; Brooks et al., 2020; Lippi et al., 2020; Urzúa et al., 2020; VeraVillarroel, 2020).

Dada la rápida expansión del virus, las primeras medidas estuvieron relacionadas con el manejo de la pandemia desde la prevención del contagio hasta la atención clínica de las personas infectadas, acciones que ya cuentan con normativas e información relativamente claras (Harapan et al., 2020). Pese a esto, las instituciones sanitarias y las sociedades profesionales no contaban con protocolos específicos para orientar el accionar de las diversas disciplinas sobre los efectos psicológicos y psicosociales derivados de la Pandemia. Ante esta situación, la Sociedad Interamericana de Psicología (SIP), a partir de la declaración de pandemia por el coronavirus (Covid-19) por parte de la Organización Mundial de la Salud (2020), emitió un mensaje a toda su comunidad para actuar con Responsabilidad, Empatía, Solidaridad y Tranquilidad frente a esta nueva situación que se presentaba como un riesgo de salud pública en las Américas y comenzó a desarrollar actividades que orientaran el quehacer de los psicólogos y las psicólogas de las Américas ante la Pandemia (Gallegos et al., 2020).

La SIP fue fundada en 1951 y constituye una organización que reúne a profesionales de la psicología en toda la región de las Américas. Desde sus orígenes ha

\footnotetext{
${ }^{2}$ Se aplica a las personas que han estado expuestas al virus, pero que no están enfermas. Implica la separación de otras personas hasta que estén fuera de la etapa de contagio.

${ }^{3}$ Se aplica a las personas que ya están enfermas, implica igualmente la separación total de otras personas hasta que se considere que están fuera de la etapa de contagio.

${ }^{4}$ Se aplica a todas las personas. Implica evitar o reducir al máximo el contacto físico, independiente del contacto o no con personas expuestas o enfermas.
} 
desarrollado múltiples actividades, como los congresos interamericanos, la publicación de una revista científica, la edición del boletín de noticias, la edición de libros, la entrega de premios, y los grupos de trabajo en diversas áreas de la psicología, entre otras (Angelini, 1979; Ardila, 1986; Consoli et al., 2015; Ferdman \& VanOss Marin, 1999; Gallegos, 2012a, 2012b, 2013; Gallegos et al., 2018).

Para hacer frente a estas y otras probables situaciones futuras, la SIP propuso la constitución del Comité "PsiCovid", con el objetivo de llevar a cabo tareas de articulación institucional y profesional para responder a las demandas suscitadas por la crisis psicosocial y de salud mental que se fueron presentando en todos los países de la región. Paralelamente, se planificó la construcción de una guía de recomendaciones que sirviera de orientación para toda la comunidad de las Américas, incluyendo especialistas, técnicos, gobiernos y ciudadanos, cuyo resultado es el que se expresa en este documento.

\section{Método}

La elaboración de este trabajo siguió los criterios técnicos acordes a la revisión sistemática de la literatura y el procedimiento metodológico de la construcción de guías y recomendaciones en el campo de la salud mental (Calva \& Gómez, 2007; Gisbert et al., 2008; Hernández et al., 2015).

Se utilizaron diferentes insumos: en primer lugar, se realizó una búsqueda y recuperación de diversas fuentes documentales en bases de datos regionales (PsycInfo, Scielo, Redalyc, y Dialnet) bajo los descriptores \#Covid-19 y \#salud mental. Paralelamente, se consultó a expertos y expertas en salud mental de diversos países y se recuperaron directrices de varios organismos nacionales, regionales e internacionales en salud mental. A partir de la abundante información recuperada, se seleccionó aquella que resultó más actualizada y que cumplía con los tópicos elegidos para la elaboración de la guía. El procesamiento de la información fue organizado alrededor de diversas preguntas, que aparecen en la sección de Resultados, las cuales abordan diferentes situaciones y problemáticas psicológicas. El resultado de esta labor derivó en la confección de un documento con recomendaciones y líneas de acción sobre la salud mental para afrontar la pandemia del coronavirus (Covid-19).

\section{Resultados}

La información recuperada y analizada fue estructurada en dos grandes dimensiones. Por una parte, se encuentran las recomendaciones generales, y por otra 
parte, se presentan las líneas de acción. Para facilitar la comprensión de la lectura, las recomendaciones fueron organizadas en torno a preguntas.

\section{Recomendaciones}

Sabemos que las situaciones de crisis y emergencias, como la presente, nos enfrentan con diversas problemáticas que deben ser abordadas con los mejores recursos disciplinares y profesionales, y para el caso, la psicología cuenta con un importante acervo de conocimientos, recursos y prácticas. A continuación, se exponen recomendaciones generales sobre diferentes temas relacionados con la salud mental y la consecuente atención psicosocial que debería proporcionarse.

Cabe advertir que las recomendaciones deberán ajustarse a las diferentes realidades e incluso contextualizarse en función del lenguaje y la diversidad cultural, por medio de referentes comunitarios o representantes de los grupos sociales. De cualquier manera, constituyen tópicos de referencias para orientar a las personas y las diferentes prácticas psicológicas que se implementen.

\section{¿Cómo actuar frente a la nueva situación?}

Es importante no negar la situación ni extremar medidas innecesarias para la situación que se tiene por delante, ya sea por medio del acopio excesivo de elementos de higiene personal y/o del hogar, como de conductas de discriminación, rechazo o estigmatización. Es probable que el miedo nos induzca a comportamientos impulsivos de rechazo o discriminación de personas, e incluso a imaginar fuentes de peligro irreales o eventuales competidores por determinados productos de higiene. Ante tales situaciones es importante tener en cuenta que las otras personas están atravesando por la misma situación y por ello se recomienda no competir ni discriminar, sino compartir y colaborar con la situación, que es comunitaria.

Las personas necesitan tener la sensación de que viven en un ambiente seguro y controlado. La inseguridad y la incertidumbre vinculadas con la pandemia, aunado a la falta de claridad sobre el fenómeno en curso, facilita la aparición de conductas de ansiedad que pueden derivar en conductas egoístas y de búsqueda individual de soluciones para problemáticas que son colectivas. Por ello, es importante fomentar la solidaridad, entendiendo que los otros no son nuestros enemigos/as, sino que incluso pueden constituirse en nuestros únicos auxilios. 
Sabemos que se han presentado situaciones de desorientación, contradicción y desorganización social frente al cúmulo de informaciones no siempre homogéneas, medidas no muy claras o en estado cambiante, que se ha acompañado en algunos casos de conductas igualmente contradictorias o desorganizadas, las cuales deben ser comprendidas como un repertorio conductual ocasionado por la actual crisis (Coetzer \& Bichard, 2020; Colegio Oficial de la Psicología de Madrid [COPM], 2020a; Lippi et al., 2020; Martín \& Muñoz, 2009; Rodríguez et al., 2006; Snider et al., 2012; Torales et al., 2020; World Health Organization [WHO], 2020a).

Algunas personas tratarán de canalizar sus temores y preocupaciones por medio de diferentes comportamientos y es probable que se presenten conductas irracionales como respuesta ante las demandas percibidas en el entorno. Sin embargo, este tipo de reacciones no son respuestas psicológicas adecuadas para afrontar la situación que vivimos, y es allí donde se deberán implementar estrategias de afrontamiento psicosocial y de conducta prosocial.

Es importante ser prudente y no generar alarma innecesaria: ni magnificar el riesgo ni desestimar su peligro para evadir la sensación de miedo. El manejo adecuado de la información oficial, pertinente y sustentada en evidencia continua es crucial para desactivar falsas alarmas, así como para calmar ansiedades y propiciar conductas más adaptativas (Centers for Disease Control and Prevention, 2020; COPM, 2020a; Fernández Millán, 2013; Martín \& Muñoz, 2009).

El miedo no sólo es una respuesta natural frente a una situación de incertidumbre y desconcierto como la que se ha instalado de manera generalizada, sino además contiene una ventaja adaptativa (Charney, 2004) que es preciso utilizar a nuestro favor, pues nos hace estar alerta y nos permite orientar la toma de decisiones, favoreciendo conductas más responsables con nosotros/as y con los demás. Por eso, resulta importante dar un debido tratamiento a las situaciones de temor e incertidumbre para que no se transformen en paralizantes y desorganizadoras (COPM, 2020a; Quezada-Schol, 2020; Urzúa et al., 2020; Vera-Villarroel, 2020).

Es importante tener en cuenta que no todas las personas reaccionarán de la misma forma frente a las eventuales situaciones de crisis que se presenten; algunos/as expondrán comportamientos no habituales, otros/as quizás sorprendan por lo inesperado de sus reacciones, habrá quienes tendrán mayor adaptabilidad a las nuevas circunstancias mientras que otros/as todo lo contrario. Es importante que aquellas personas que han presentado un mejor afrontamiento de las situaciones de crisis sirvan de referencia de 
consulta y ayuda y puedan transmitir un mensaje de objetividad, tranquilidad y optimismo.

\section{¿Cómo actuar frente a la expresión de síntomas psicológicos?}

Es importante que cuando una persona presenta síntomas de ansiedad, nerviosismo continuo, tensión sostenida, sensación de peligro inminente, preocupación excesiva, dificultad para interesarse por otros asuntos, problemas para conciliar el sueño, aumento del ritmo cardiaco, sudoración, respiración acelerada, temblores sin causa justificada o síntomas de pánico, trate de identificar aquellos pensamientos o ideas que están causando sus malestares, los acepte, recupere la calma y pueda darle una expresión adecuada a las emociones asociadas (COPM, 2020b; Fernández Millán, 2013; Lorenzo Ruiz, Díaz Arcaño, \& Zaldívar Pérez, 2020; Martín \& Muñoz, 2009; Rodríguez et al., 2006; Snider et al., 2012; Vera-Villarroel, 2020; WHO, 2020). Si es posible sería beneficioso compartirlas con alguien de su entorno próximo o de forma virtual con un familiar, amigo o vecino de confianza.

Cuando las demandas sobrepasan los recursos psicológicos de las personas, existen diferentes cursos de acción que ofrecen beneficios a corto o a largo plazo. Las estrategias de afrontamiento centradas en el problema o en las emociones, por ejemplo, pueden ser muy útiles, ya sea porque permiten buscar soluciones directas a la problemática o regular las respuestas emocionales a la misma (Roth \& Cohen, 1986). Practicar un ejercicio de cuestionamiento de la situación que atemoriza por medio de la búsqueda de información calificada, contrastando evidencia empírica y datos fiables es una estrategia útil para reevaluar nuestra percepción del riesgo y disminuir temores. También puede resultar muy útil descentrar el foco de atención y buscar temas alternativos de conversación o actividad como un tipo de estrategia afrontamiento evitativa centrada en las emociones para obtener efectos positivos temporales. Incrementar el apoyo social puede ser fundamental, ya sea acudiendo a personas que pueden generar tranquilidad o informando a algún familiar, amigo/a o vecino/a de confianza cuando uno se encuentre solo/la. Otras alternativas beneficiosas para el manejo de los síntomas consisten en escribir o mantener un diario con sus pensamientos (Baikie \& Wilhelm, 2005), realizar ejercicios, o caminar (Wipfli et al., 2008), y hacer ejercicios de relajación, respiración o meditación (Sedlmeier et al., 2012). Cada persona debe definir 
las estrategias y acciones que mejor se adecúen o le ayuden para afrontar la situación y superar los síntomas o manifestaciones asociadas con esta situación.

También resulta importante recuperar las habilidades, capacidades y destrezas con las cuales se afrontaron situaciones similares en el pasado. Desde una perspectiva de proceso, las personas cuentan una capacidad de resiliencia inherente que pueden incrementar y utilizar a su favor para adaptarse y superar la pandemia del Covid-19 (Rosenberg, 2020).

Cuando nada de esto resulte y los síntomas persistan o se agraven, se deberá buscar ayuda especializada en los canales oficiales habilitados o en consulta particular. Una forma de valorar la necesidad de recurrir a un/a profesional es cuando la angustia se vuelva debilitante o insoportable, cuando aparezcan pensamientos de muerte, cuando se dificulte establecer una discriminación entre hechos reales e imaginarios, cuando se generen conductas de riesgo para sí mismo o los demás, entre otros. En cada país diversas instituciones y sociedades profesionales están ofreciendo servicios de telepsicología (asistencia psicológica virtual) que pueden consultarse para atender esta situación.

\section{¿Cómo afrontar situaciones de cuarentena y de distanciamiento saludable?}

La principal recomendación para atenuar la propagación del contagio del Covid19 por medio del distanciamiento físico ha involucrado cambios en el estado de ánimo de las personas y sus entornos familiares, no sólo porque se ven recluidas en sus domicilios, planteándose nuevas condiciones de hábitat, sino además porque se ha interrumpido su cotidianeidad. Es importante tener presente que una de las características humanas más importantes es la necesidad de establecer vínculos con otros, y precisamente la fórmula más eficaz para combatir la extensión del contagio atenta contra esa necesidad primordial.

Si bien el término "distanciamiento social" ha sido reemplazado en algunos lugares por el de "distanciamiento físico", es preferible alentar la idea de un "distanciamiento saludable", que no implique desconexión social, emocional o interpersonal. La interacción social directa que tenemos actualmente $-\mathrm{y}$ que continuaremos en el contexto de la pospandemia - incorpora diferentes medidas sanitarias como lo son el uso de paneles acrílicos separadores, la sanitización de los espacios de proximidad, así como elementos de higiene y protección personal (e.g., cubrebocas) a las que deberemos acostumbrarnos. En contextos de interacción social virtual es posible sortear mejor el distanciamiento con otros, mientras guardamos muchas 
de las medidas sanitarias impuestas. Los medios virtuales de comunicación e interacción social permiten obtener apoyo social y mantener el bienestar psicosocial (Guil-Bozal et al., 2018). Para evitar el aislamiento y sus efectos negativos es necesario buscar alternativas para estar comunicados con amigos/as y compañeros/as de trabajo, de igual manera que con los otros integrantes del seno familiar extendido, sobre todo con los niños y niñas.

Lógicamente la reclusión de las familias en sus respectivos domicilios ha implicado la necesaria adecuación de las normas de convivencia y las pautas de interacción. Naturalmente surgen conflictos de convivencia que deben ser abordados con posiciones constructivas y colaborativas, respetando los tiempos de introspección de cada persona. Cuando es posible es beneficioso mantener hábitos familiares usuales, rutinas de trabajo virtuales, estudio programado y horarios de descanso, juego, ejercicio y ocio. La higiene personal y familiar es de capital importancia, así como el establecimiento de una dieta balanceada y nutritiva, e hidratación frecuente. Las actividades manuales y el ejercicio físico son muy favorables para el control de la ansiedad y cumplen un papel de distracción. Es suma, es importante reestructurar una planificación familiar de acuerdo con las características y los tiempos de las personas involucradas (COPM, 2020a, 2020b; Inter-Agency Standing Committee [IASC], 2020; Organización Panamericana de la Salud/Organización Mundial de la Salud [OPS/OMS], 2020).

La cuarentena y cualquier tipo de aislamiento causan malestar generalizado, estrés, irritabilidad, ansiedad, desánimo, enojo, frustración, aburrimiento, miedo, desesperanza y sus manifestaciones pueden persistir durante el tiempo de pandemia o reaparecer posteriormente al restablecimiento de las actividades (Brooks et al., 2020). Es muy probable que se presenten alteraciones del sueño y del ritmo circadiano, evidenciándose a través de la pérdida de referencia temporo-espacial. Será importante no automedicarse (López-Cañón \& Pérez-Acosta, 2020), y resultará más aconsejable plantearse diversas estrategias de distracción y/o ocupación, como el ejercicio físico, lectura, música, películas, etc.

Es importante afrontar el periodo crítico con optimismo y como un resguardo necesario de la salud individual, familiar y colectiva. Puede ser un momento propicio para replantear objetivos personales y familiares. Igualmente se recomienda aprovechar la reclusión para destinarla a aquellas actividades o proyectos que generalmente suelen postergarse por falta de tiempo, así como también aprovechar para reconectar o profundizar los vínculos con las personas del entorno familiar. 


\section{¿Cómo informarse adecuadamente?}

Circula mucha información falsa y contradictoria, y por ello es vital acudir a los organismos oficiales. Una de las formas para combatir el temor es recurrir a fuentes de información oficiales y personas confiables. Resulta importante evitar la sobreexposición informativa y no replicar información innecesaria (IASC, 2020; Limaye et al., 2020; OPS/OMS, 2020). Es preferible replicar memes, chistes y humoradas que apunten a desdramatizar la situación, por supuesto, sin generar descalificaciones ni herir susceptibilidades. El humor siempre es un buen recurso para tramitar situaciones traumáticas (Garrick, 2006).

Por otra parte, es importante tener en cuenta que el acceso a la información, principalmente de primera necesidad como el sanitario y los servicios de asistencia psicológica, no siempre está garantizado para toda la población; por ello resulta primordial plantear estrategias coordinadas con los organismos gubernamentales para idear formas más eficaces y/o alternativas de comunicación a los sectores más vulnerables.

\section{¿Cómo actuar con los niños y las niñas?}

Es importante tener en cuenta que la mejor forma de proteger y cuidar a los niños/as es a partir de la responsabilidad de los adultos con su propia salud, el manejo de la información pertinente, la seguridad y la sencillez en la transmisión de las normas de higiene y la prevención del contagio. Existen varias recomendaciones generales que podrán tomarse en consideración para manejar la situación con los niños y niñas (COPM, 2020c; IASC, 2020; OPS/OMS, 2020).

Si los adultos revisan continuamente sus celulares o la televisión para obtener las últimas actualizaciones sobre el coronavirus y reaccionan con preocupación, lo más probable es que los niños se hayan percatado de dicho comportamiento y aprendan a reaccionar con miedo y ansiedad por modelaje y aprendizaje vicario (Chorpita et al., 1996). Por ello es necesario hablar con calma y transmitir tranquilidad.

No es recomendable que se prohíba hablar de la temática. Por el contrario, se recomienda que se hable de la misma cada vez que sea necesario, pero sin exponerlos/as a una saturación de la información. Se recomienda elegir un momento adecuado para hablar con los niños y niñas sobre el coronavirus. Idealmente debería ser un momento del día que no sea muy tarde y donde puedan concentrarse, sin que les afecte el sueño debido 
a los posibles temores sobre la información proporcionada o la percepción que están teniendo del entorno.

Es importante brindar el tiempo necesario para que los niños/as compartan sus propios temores. Una forma de atenuar esos temores es proporcionándoles algún grado de control sobre la situación. Por ejemplo, induciendo ciertas pautas de comportamiento que exige la situación presente como lavarse las manos, no tocarse los ojos con las manos, dormir a la hora adecuada, entre otros. En general, como se ha sostenido anteriormente, los padres son los modelos de conductas que los niños/as suelen adoptar para sí mismos, de ahí la importancia señalada más arriba del comportamiento adecuado de los padres.

También es importante hablarles con sinceridad, no ocultar información vital, corregir ideas erróneas, responder a las dudas, temores y manifestaciones conductuales. Se recomienda brindar seguridad y confianza en que la problemática de salud se está abordando adecuadamente y que se solucionará prontamente con una eficaz prevención. Además, hay que trasmitir que la problemática no conlleva mayores riesgos de salud si se aplican adecuadamente las normativas sanitarias.

Si los niños/as realizan preguntas a las cuales no se puede dar una respuesta por desconocimiento, se deberá buscar información clara y veraz al respecto. Se aconseja nuevamente la utilización de fuentes de informaciones oficiales y confiables. Existen diferentes recursos didácticos especialmente elaborados para explicarles a los niños y las niñas la problemática del coronavirus (Algarín et al., 2020; Bloj, 2020; Molina, 2020).

Resulta muy útil la explicación de la información por medio de representaciones gráficas, sean prediseñadas o elaboradas conjuntamente con ellos y ellas. Las actividades lúdicas con los niños y las niñas siempre son excelentes recursos para transmitir ideas, informaciones, comportamientos y acciones, además de que se trata de una actividad placentera, distendida y reforzante de emociones positivas (Fancourt \& Finn, 2019).

Los niños y niñas suelen preocuparse más por su familia y sus amigos que por sí mismos. Si escuchan reiteradamente que sus abuelos/as tienen más probabilidades de enfermarse de gravedad, pueden asustarse, por eso se recomienda una adecuada comunicación telefónica con ellos para aliviar el posible temor.

No es necesario neutralizar absolutamente todo sentimiento de miedo, ya que es normal sentirse atemorizado en determinados momentos de la vida. Es importante poder reconocer estos sentimientos y darles la atención adecuada, sabiendo que se trata de una situación transitoria, y que en algún momento la vida volverá a la normalidad, todo lo 
cual contribuirá a desarrollar la capacidad de afrontar situaciones adversas (resiliencia) (Penagos-Corzo et al., 2019; Rosenberg, 2020).

Una atención especial merecen los niños y las niñas institucionalizadas, quienes se encuentran fuera del círculo familiar y por ende constituyen un grupo vulnerable, que deberá asistirse de forma especializada. Aun cuando las sugerencias expresadas más arriba se puedan aplicar a este grupo, deberán planificarse acciones de contención emocional, transmisión adecuada de la información y cuidados de higiene especial.

\section{¿Cómo actuar con adolescentes?}

Es importante tener en cuenta que los/as adolescentes cuentan con sus propias redes sociales y medios de socialización y comunicación, donde la información divulgada puede ser errónea (IASC, 2020; OPS/OMS, 2020); por ello, sería ideal aprovechar esta situación para habilitar canales de diálogos más reflexivos y críticos. Quizás sea un buen momento para conectar o reconectar ciertos vínculos con los/as adolescentes que por la propia dinámica de la vida contemporánea se fueron diluyendo.

Si bien se espera una actitud colaborativa con la situación y un compromiso mayor con el cumplimiento de las normativas que se implementen, es posible que también se manifiesten comportamientos de rebeldía, transgresión, fastidio, malhumor o agresividad. Hay que tener en cuenta que el sentimiento de omnipotencia e invulnerabilidad frente a las amenazas que son características de los adolescentes (Santrock, 2019) puede conducir a resistencias y/o transgresiones de las normativas de asilamiento y confinamiento en el hogar. Estas manifestaciones no sólo hay que comprenderlas como características prototípicas de la edad, sino además en el contexto del cambio en las condiciones de habituación y socialización, y las consecuentes restricciones que se les impongan.

Frente a estas eventuales manifestaciones es importante apuntar a la necesaria responsabilidad con la propia salud, la de su familia y la de sus amigos/as y compañeros/as, la adecuada comprensión de la problemática social, la negociación de actividades en la situación presente y futura, y el establecimiento de ciertos acuerdos sobre las tareas, los roles, los tiempos, las actividades, entre otros. Siempre será importante respetar los tiempos de cada cual, más allá de las actividades o labores que se planteen colectivamente. 


\section{¿Cómo actuar con los adultos mayores?}

Los adultos mayores representan la población más vulnerable y la mayoría de ellos son conscientes de esta situación, por lo que es muy importante brindar asistencia psicológica que apunte a calmar las ansiedades y los temores lógicos. También es importante brindarles espacios de información donde puedan plantear sus dudas respecto de la enfermedad y sus posibles complicaciones (IASC, 2020; OPS/OMS, 2020).

Es importante tener en cuenta que se trata de una población bastante heterogénea en cuanto a condiciones de salud, situaciones habitacionales y posibilidades de asistirse por sí mismos, por ello deberán elaborarse estrategias diferenciadas para cada conjunto de situaciones.

Las medidas de prevención e higiene deben ser muy estrictas con esta población. Es probable que muchos precisen de la asistencia de familiares o vecinos, por eso el apoyo y la solidaridad de la comunidad resulta crucial para este grupo social. Sería ideal que se pudieran desplegar estrategias de relevamiento barrial/comunitario para contar con un mapa de las necesidades específicas, con la consecuente designación de referentes que se ocupen de asistir a las personas que lo requieren.

\section{¿Cómo actuar frente a personas con discapacidad?}

La población de personas con discapacidad presenta características similares a la de los adultos mayores en cuanto a la necesidad de asistencia, los requerimientos de higiene y la gran heterogeneidad de situaciones que deberán contemplarse, tanto en los hogares como en las instituciones donde se encuentren hospedadas o con actividades laborales. De igual forma, representan un grupo de gran vulnerabilidad y suponen en muchos casos una carga extra para las familias y/o sus cuidadores a la hora de establecer una adecuada planificación de las tareas y las nuevas normas de convivencia en el seno familiar (Martínez et al., 2016).

Es importante estar alerta frente a los sentimientos de desconcierto y angustia que puedan emerger debido a la pérdida de las rutinas y los cambios que se introduzcan en los entornos de convivencia. La restricción de ciertos hábitos cotidianos puede dar lugar diversas manifestaciones de agresividad y malestar generalizado, para lo cual se deberá idear estrategias de sensibilización y preparación cuando sea posible.

Un especial cuidado deberá tenerse respecto de aquellas personas que afrontarán este periodo de asilamiento en instituciones públicas o particulares, de modo que puedan 
garantizarse sus derechos, las condiciones necesarias de asistencia, higiene y contención emocional. Será importante favorecer un trato igualitario y no discriminativo en cuanto al acceso a la información y la disponibilidad de los recursos sanitarios.

\section{¿Cómo actuar con personas afectadas por problemas de salud mental?}

Otro grupo de gran vulnerabilidad son las personas que están cursando algún tipo de padecimiento mental. Diferentes medidas de aislamiento y reclusión pueden afectar el estado de ánimo de las mismas, con el consecuente agravamiento de los diferentes cuadros psicopatológicos (Huarcaya-Victoria, 2020). En consecuencia, las familias y las instituciones que estén al resguardo de estas personas deberán ofrecer una asistencia y un monitoreo específico a cada uno de ellas.

\section{¿Cómo actuar con personas en situación de calle?}

Desde luego, este grupo de personas son altamente vulnerables a todo tipo de discriminación, maltrato, falta de consideración y acceso a las condiciones básicas de higiene que hoy se están planteando como medidas preventivas de contagio. Por tanto, será importante instrumentar acciones específicas desde los organismos estatales para velar por el cuidado de estas personas y garantizar un tratamiento digno frente a sus condiciones y necesidades particulares.

\section{¿Cómo actuar como padres?}

Es importante que los padres o referentes del hogar puedan elaborar, conjuntamente con los miembros del entorno, una rutina de actividades, tareas y asignación de roles mientras dure el aislamiento, ya que puede favorecer una mejor adaptación a la situación nueva (IASC, 2020; OPS/OMS, 2020). Siempre será ideal si esta organización se plantea a modo de trabajo en equipo, con la aceptación y el compromiso de cumplimiento de las normas que se establezcan.

Es recomendable que se pauten tiempos para cada actividad, como por ejemplo el aseo del hogar, el tiempo de conectividad en Internet, la actividad física, las tareas de la escuela o universidad, entre otros. En general, se recomienda aprovechar esta instancia transitoria para habilitar otras formas de convivencias y estrechar los vínculos familiares. 
Es importante tener presente que los padres o referentes siempre serán la autoridad del hogar, y de ellos dependerá el mantenimiento de la organización y la planificación familiar. Sin embargo, puede darse el caso que algunos de los padres o ambos deban ausentarse, y para ello también deberá planificarse la suplencia de roles, el mantenimiento de la organización y los compromisos propios del hogar.

Frente a la emergencia de situaciones conflictivas, se recomienda no reprimirlas con autoridad, sino más bien habilitar un tratamiento adecuado a través de la palabra y el entendimiento mutuo. Cuando ello no sea posible, debido a la energía volcada en el conflicto, será importante tomar distancia y retomarla en un momento posterior. Será muy importante respetar los tiempos de introspección de cada sujeto, su malhumor, desánimo o contradicción; tales sentimientos pueden afectar a cualquiera de los miembros de la familia, independiente de sus roles o jerarquías.

\section{¿Cómo orientarse con las personas en confinamiento carcelario?}

Las situaciones carcelarias, con sus diferentes realidades y condiciones en los países de las Américas, exponen una problemática particular desde el punto de vista de la salud física y mental. El confinamiento al que están sometidos los/as reclusos/as no los/as vuelve inmunes al contagio ni tampoco menos propensos a consecuencias psicológicas diversas. Por tanto, será importante administrar el servicio psicológico adecuado para estas poblaciones, con especial foco en las poblaciones vulnerables, como las personas con enfermedades predisponentes y adultos mayores (National Institute for Health and Care Excellence, 2017; WHO, 2020b).

\section{¿Cómo enfrentar la crisis humanitaria de los refugiados, migrantes y desplazados?}

La propagación del contagio por el Covid-19 ha profundizado la crisis humanitaria de miles de personas que se han visto desplazadas de sus lugares de orígenes y/o se encuentran en situación migratoria en diferentes lugares de las Américas. Estas poblaciones son altamente vulnerables y es muy probable que dichas situaciones estén siendo descuidadas, no sólo en cuanto a los recursos básicos de higiene y salubridad para hacer frente a la extensión del contagio, sino también en cuanto a los recursos de apoyo emocional y psicosocial de los individuos, grupos y familias (IASC, 2009; WHO, 2015).

Las situaciones traumáticas, la condición de desamparo, el choque cultural, la no pertenencia, la sensación de alienación, la desrealización personal, el peligro constante y 
la falta de expectativas son algunas de las vivencias que deberán ser abordadas desde el apoyo emocional y la contención psicosocial. Afortunadamente, si bien estas poblaciones suelen estar expuestas a grandes padecimientos psicológicos, también suelen ser muy resilientes (COPM, 2016; Rosenberg, 2020).

\section{¿Cómo orientarse respecto de la sexualidad y las diversidades inherentes?}

La sexualidad es un componente importante de la salud mental y, por tanto, debe considerarse como un asunto de salud pública, sin prejuicios ni tabúes. La misma debe ser orientada por especialistas atendiendo a la diversidad sexual y las múltiples identidades de género que se hoy se reconocen. El respeto a la identidad sexual, la no discriminación y la no estigmatización deberán ser preceptos que orienten los enfoques preventivos y las asistencias que se realicen.

Inevitablemente las medidas de distanciamiento saludable conllevan a la distancia sexual física, y por ello se aconseja la utilización de medios virtuales, siempre resguardando la privacidad de los/as practicantes. Las prácticas de satisfacción sexual más seguras son la masturbación (y otras prácticas no penetrativas), las relaciones sexuales con personas del círculo íntimo, evitando el sexo grupal y los encuentros eventuales. Es preciso tener en cuenta que debido a los temores provocados por el contagio del Covid-19 muchas personas se nieguen a tener relaciones sexuales (Pineda Roa, 2020).

\section{¿Cómo orientarse en situaciones de duelo?}

Las situaciones de duelo son muy diversas e implican tanto a las personas que cursan alguna enfermedad terminal como a grupos y personas que se enfrentan con la pérdida de familiares, amigos/as o cuestiones vitales de su entorno cotidiano. Además es preciso tener en cuenta que la muerte es una experiencia que difiere culturalmente de un contexto a otro. Sin embargo, cualquier situación dolorosa o trágica que implique alguna pérdida, necesariamente conlleva un proceso de afrontamiento psicológico. Cuando sea necesario, según la dificultad de afrontamiento, deberá recurrirse a la atención psicológica especializada para tramitar el proceso de duelo (Worden, 1997).

La presente situación impone una modalidad de abordaje diferente ante las situaciones de duelo, ya sea por la asistencia virtual o por las condiciones en las cuales las personas se enfrentan con la pérdida, principalmente por la imposibilidad de 
acompañamiento previo, la ausencia de funerales o rituales habituales. En estos casos será importante dar lugar al proceso de simbolización de la pérdida a través de medios alternativos a los habituales, como la escritura de una carta, los homenajes de despedida a través de la web y redes sociales, la elaboración de un álbum de recuerdos fotográficos, dibujos con los más chicos, y demás actividades que ayuden a canalizar las emociones asociadas al duelo.

Es factible que se presenten sentimientos de tristeza, culpa, melancolía, soledad, frustración, enfado, irritabilidad, entre otros. También conductas desadaptativas, automedicación, consumos de sustancias, conductas de riesgo, etc. (Pérez-Acosta, 2020). La asistencia en la elaboración del duelo deberá tener en cuenta, entre otras variables, las diferentes edades, la capacidad de procesamiento de la información de cada persona y los recursos de afrontamiento. Será importante no sofocar el dolor, sino más bien habilitar un tratamiento adecuado de acuerdo con las circunstancias y las posibilidades de cada persona o grupo de individuos.

\section{¿Cómo acompañar al personal de la salud y otras actividades esenciales?}

Es importante contextualizar que el personal de la salud y aquellas asignadas a las actividades esenciales, no sólo se encuentran expuestas al contagio del virus, sino también a una importante carga emocional por las actividades que desarrollan y las situaciones que enfrentan, muchas de las cuales están más allá de sus posibilidades (Lorenzo Ruiz \& Guerrero, 2017; Sim \& Chua, 2014). Además de las medidas de seguridad e higiene que deben cumplir, también resulta necesario que cuenten con el debido acompañamiento emocional y psicosocial. Si bien resulta significativo realizar el debido reconocimiento a su labor, no sería recomendable exagerar su heroísmo, ya que podría crearse una falsa imagen de sus capacidades, llevándolos a asumir responsabilidades desmedidas que pongan en riesgo su propia salud.

La exposición reiterada y sostenida en el tiempo a diversas situaciones estresantes y traumáticas desde el punto de vista bioético puede acarrear importantes daños psicológicos en esta población, sea en el presente inmediato como en el futuro. En el caso del personal de la salud, aun cuando se trata de una población dedicada a la atención de situaciones extremas, ello no los vuelve inmunes a los padecimientos psicológicos. Es probable que se presenten sensaciones de impotencia, falla, temor, decepción, fatiga, incertidumbre, angustia, shock emocional, confusión, incapacidad para descansar y 
sobreexigencia. Para prevenir algunas de estas conductas, pensamientos y sentimientos nocivos, será beneficioso realizar actividades de desensibilización, relajación, distracción y reuniones de equipo cuando finalicen las jornadas laborares.

\section{¿Cómo orientarse frente a los abusos, la violencia intrafamiliar y la violencia de género?}

Resulta importante indicar que los abusos, la violencia en el hogar y la violencia de género lamentablemente son situaciones arraigadas en las sociedades en las que vivimos. Es decir, son preexistentes a la realidad del Covid-19. No obstante, la imposibilidad de circulación debido a las medidas de confinamiento puede volverse una trampa mortal para muchas personas. Por tanto, se vuelven necesarias todas las medidas preventivas, de acompañamiento, seguimiento y asistencia emocional y psicosocial que se puedan establecer desde los organismos estatales y equipos profesionales. La asistencia psicológica virtual, las campañas de sensibilización, los códigos de alerta, la preparación de los refugios seguros, el involucramiento de la sociedad, entre otros, son recursos valiosos para canalizar las demandas a cada situación.

Mujeres, niños/as y adultos mayores son las víctimas más frecuentes de la violencia intrafamiliar (United Nations, 2020). Existen diferentes manifestaciones de la violencia y no siempre son fácilmente reconocidas por las personas que las sufren: física, verbal, patrimonial, moral, sexual, maltrato, desvalorización, menosprecio, entre otras. Este tipo de situaciones conduce a un daño psicológico en las personas y pueden resultar incapacitantes para el desenvolvimiento de actividades cotidianas. Las personas que se encuentran expuestas a cierto tipo de violencia, que no necesariamente implica la agresión física, pueden quedar ligadas a un circuito difícil de manejar y escapar, precisamente por la naturalización de la situación en la que viven (Aiquipa Tello \& Canción Suárez, 2020). De ahí la importancia de una asistencia psicológica adecuada y precisa, debido a que las medidas de reclusión en los hogares pueden atrapar aún más a las víctimas en el circuito de la violencia.

\section{¿Cómo orientarse frente los consumos problemáticos?}

Las diversas situaciones a las que se enfrentan las personas por las medidas sanitarias implementadas y la carga emocional inherente, pueden llevar a un aumento exagerado del consumo de diversas sustancias, lo cual podría ser muy dañino para la salud 
de estas. Cabe situar que el consumo exagerado de alcohol, el tabaco, las drogas y los consumos problemáticos en general representan importantes problemáticas de salud pública y para muchas personas suponen una problemática preexistente a la pandemia, pero esta nueva situación puede agravarlos (Pautassi \& Bonino, 2020; Rojas-Jara, 2019, 2020).

El uso de sustancias puede ser lúdico o experimental, pero también convertirse en una conducta adictiva y/o descontrolada. La valoración de la situación cambia cuando el consumo se transforma en un problema para las personas y su entorno. El consumo de diferentes sustancias conlleva a una alteración de las experiencias subjetivas de las personas y puede amplificar diferentes tipos de emociones y conductas: alegría, tristeza, rabia, evasión, agresión, depresión, manía, autolesión y/o violencia. Precisamente, por tratarse de cuestiones que involucran fundamentalmente el equilibrio de las emociones y ponen en riesgo el comportamiento individual y colectivo, es importante que se desplieguen acciones de asistencia psicológica especializadas.

\section{¿Cómo orientarse ante la desigualdad social y la discriminación?}

La pandemia del Covid-19 está afectando en forma contundente a los grupos minoritarios y las personas más pobres y vulnerables de las naciones. Estas son las personas que están sufriendo de manera desproporcionada en todo el mundo, incluidos los países con más alta tasa de desarrollo. Sus padecimientos se deben a la dificultad para satisfacer sus necesidades fundamentales de alimentación, la falta de acceso a los recursos básicos de salud, las condiciones de hacinamiento, el incremento del desempleo, la insuficiencia de la asistencia gubernamental, etc. Estas desigualdades se han constatado en todos los países y continentes, y la situación tiende a agravarse aún más durante el curso de la pandemia. Muchas personas no cuentan con la posibilidad de aislarse ni poseen los medios para acceder a los recursos de protección sanitaria.

Las y los profesionales de la psicología, así como los servicios de salud mental deben prestar especial atención a las personas de bajos ingresos pues son las más afectadas por la pérdida temporal de ingresos y muestran niveles más elevados de estrés y depresión (IASC, 2020; OPS/OMS, 2020). También resulta importante prestar especial atención a los problemas de discriminación, ya sea en la atención diferenciada o de baja calidad de los servicios, como en la estigmatización de las personas mayores, de diferentes culturas o nacionalidades, especialmente aquellas a quienes se responsabilizan 
de ser causantes de la pandemia. Tales personas pueden estar experimentando los efectos psicológicos de la discriminación, además de las consecuencias por la enfermedad del Covid-19. Es importante que los y las profesionales de la psicología aboguen por un trato digno, el respeto a la identidad y acorde a los principios de los derechos humanos de todas las personas sin discriminación ni trato diferenciado.

\section{¿Cómo adecuar las prácticas profesionales de atención psicológica?}

Se recomienda evitar la actividad presencial y continuarla por medios virtuales, tanto en las intervenciones individuales como grupales. De no ser posible, según las circunstancias, es necesario acatar las recomendaciones sanitarias como en cualquier otro ámbito de interacción social: mantener distancia, evitar el contacto físico, utilizar mascarillas o cubrebocas, lavarse las manos, usar alcohol en gel, limpieza y desinfección del espacio común y espaciar los turnos de las consultas para limitar los contactos entre pacientes.

Tanto los dispositivos de acompañamiento y orientación psicológica como las prácticas psicoterapéuticas virtuales (Telepsicología) deberán seguir todas las normativas éticas y deontológicas de la profesión psicológica, y los protocolos de actuación normalizados en cada país/región (Joint Task Force for the Development of Telepsychology Guidelines for Psychologists, 2013; COPM, 2020c).

\section{¿Cómo colaborar profesionalmente con la situación problemática?}

Es importante que los psicólogos y las psicólogas, así como las instituciones profesionales que los/as nuclean desarrollen acciones de información, atención y contención de las situaciones que se presenten durante y posterior a la contingencia sanitaria del Covid-19. La presencia de los psicólogos y las psicólogas en los medios masivos de comunicación es crucial para llevar un mensaje de tranquilidad a la población y desactivar la circulación de informaciones no sustentadas en conocimientos fundamentados.

Es preciso que la comunidad profesional y las instituciones psicológicas respectivas contribuyan con los mejores recursos disponibles para afrontar esta problemática transnacional de salud pública. Debido a que no es posible prever cuánto durará este evento sanitario, ni los múltiples efectos y repercusiones sobre la salud mental de la población, es importante estar preparados adecuadamente, a través de la 
capacitación específica, la conformación de equipos técnicos, y fundamentalmente, el cuidado de la propia salud de los agentes y profesionales involucrados en las tareas en terreno.

Es igualmente necesario continuar desplegando acciones de monitoreo, observación e investigación del comportamiento individual y social frente a esta situación específica, para recabar aquellos datos esenciales que luego permitan tomar mejores decisiones. No se trata de que no se posea conocimientos al respecto, pero resulta primordial implementar estrategias de observación y seguimiento de casos específicos para establecer un mapa de la salud mental global de la población, sobre el cual será posible sustentar acciones de atención a las necesidades psicológicas y psicosociales que se presenten.

\section{¿Cómo colaborar profesionalmente con los gobiernos nacionales?}

La psicología es una profesión que cuenta con un acervo de conocimientos y prácticas para aportar a esta situación crítica que atraviesa la salud pública, no sólo desde el plano individual, grupal y social, sino también desde la elaboración, el diseño y la puesta en práctica de políticas, programas y acciones de contingencia sobre la salud mental de la población.

Por ello se invoca a los gobiernos a recurrir a los/as profesionales competentes y las instituciones representativas para continuar ideando acciones que permitan hacer más eficaz las medidas sanitarias que se han implementado, y se insiste en la necesidad de abordar la salud en sus planos físico, biológico, emocional y psicosocial. Corresponde a las entidades gubernamentales proveer los recursos, insumos y condiciones necesarias que favorezcan la intervención de los profesionales de la psicología en los planes desarrollados.

Asimismo, resulta imprescindible indicar que la presente situación de crisis no sólo requiere la implementación de estrategias específicas de promoción y atención de salud mental mientras continua el evento, sino también posterior a la normalización de las actividades debido a los efectos postraumáticos y de cambios en las relaciones humanas que puedan presentarse. 


\section{Líneas de acción}

Las acciones representan un conjunto de lineamientos que sirven de guía para las diversas actividades que se están realizando por parte de las y los trabajadores sanitarios, profesionales de la psicología y organizaciones psicológicas. También representan un llamado a la ciudadanía y los gobiernos nacionales a nutrirse de los conocimientos y recursos que tiene la psicología como disciplina y profesión para afrontar los desafíos de la presente pandemia y sus posteriores consecuencias.

La primera acción implica el reconocimiento y la aceptación de las recomendaciones sanitarias oficiales, emanadas por los organismos nacionales competentes, dando especial tratamiento a las diferentes medidas que se implementen para el cuidado de la salud colectiva. Conjuntamente con ello, es primordial orientarse por las informaciones oficiales de los organismos competentes en salud y evitar información de dudosa procedencia, privilegiando la información científica y basada en evidencia. La infodemia (cantidad excesiva de información que no permite llegar a una solución del problema) puede reducir la confianza y la probabilidad de seguir las recomendaciones correctas.

La segunda acción invoca a la responsabilidad social, la solidaridad comunitaria y la necesaria tranquilidad que requiere la presente situación de pandemia y su posterior derivación. Esto implica sostener un comportamiento individual y social acorde a las necesidades y requerimientos de la salud colectiva y el acatamiento de las normativas sanitarias. Desalentar conductas que pongan en riesgo la salud individual, familiar y colectiva. Mantener la calma y promover conductas que aumentan el bienestar personal, familiar y social.

La tercera acción envuelve la necesidad de que las instituciones profesionales de psicología conformen equipos técnicos de acompañamiento, orientación y atención psicológica, y su respectiva capacitación especializada, sea presencial o virtual, para canalizar las consultas emergentes y la elaboración de estrategias psicoprofilácticas. Resulta primordial que las y los profesionales de la salud mental puedan llevar a cabo estrategias de atención psicológica para atenuar los impactos emocionales provocados por la extensión de la pandemia y las consecuentes medidas sanitarias de aislamiento, entre otros fenómenos eventuales de crisis psicosocial.

La cuarta acción insiste en la importancia de llevar a cabo prácticas psicológicas con sensibilidad cultural, respetando la identidad de las personas, sensible a las 
desigualdades sociales, sin discriminación por razones de procedencia, sexualidades y condiciones socioeconómicas. Desde luego, estas prácticas deberán sustentarse en los principios fundamentales de los derechos humanos, y los principios éticos y deontológicos de la profesión psicológica.

La quinta acción convoca a los gobiernos y sus respectivos órganos de decisión a proveerse de los recursos disciplinares y profesionales de la psicología para abordar de manera integral la presente situación de crisis sanitaria internacional. La psicología posee herramientas para llevar a cabo medidas tanto preventivas, en la implementación de conductas de autocuidado y de medidas de contención, como en el diagnóstico, asesoramiento y atención psicológica, así como en la comunicación de riesgos y el desarrollo de estrategias de participación comunitaria, necesarias para afrontar esta pandemia. Resulta primordial que la salud individual y colectiva deba abordarse integralmente, es decir, en sus dimensiones física, biológica, psicológica y social.

\section{Conclusión}

La aparición del Covid-19 ha implicado innumerables desafíos a nivel global y todavía no se pueden estimar con certeza las múltiples consecuencias que tendrá en el medio y largo plazo. Sin embargo, ya contamos con una medida de los impactos y las necesidades que deberán atenderse. La región de las Américas cuenta con diversas capacidades de afrontamiento institucional y profesional en el campo psicológico y sería recomendable que las mismas sean aprovechadas estratégicamente por los respectivos gobiernos nacionales. La elaboración de la presente guía es el reflejo sumario de todas esas capacidades y recursos que posee la psicología.

Dada le diversidad cultural, lingüística y geopolítica de los países de las Américas, se espera que esta guía sirva de marco general para llevar a cabo acciones de prevención, asistencia y abordaje de la salud mental y la promoción del bienestar psicosocial de la población. Se espera también que, con la apropiada adecuación a los diferentes contextos socioculturales, sea de utilidad para orientar a la ciudadanía y las prácticas profesionales de los psicólogos y psicólogas. Por último, que sea una herramienta de consulta para la toma de decisiones en materia de salud mental tanto para las organizaciones psicológicas como para los organismos gubernamentales en salud pública de los diferentes países de las Américas. 


\section{Referencias}

Aiquipa Tello, J. J., \& Canción Suárez, N. M. (2020). Mujeres supervivientes de violencia de pareja: factores y procesos psicológicos implicados en la decisión de permanecer o abandonar la relación. Avances en Psicología Latinoamericana, 38(1), 189-202. http://dx.doi.org/10.12804/revistas.urosario.edu.co/apl/a.7139

Algarín, G., Torres, H., Meléndez, L. \& Santos, A. (2020). ¡Achú! Libro didáctico para explicar a chicos el coronavirus. https://prsciencetrust.org/wpcontent/uploads/prpht/Activity-book-coronavirus-5.pdf

American Psychological Association (2020). Pandemics. https://www.apa.org/practice/programs/dmhi/research-information/pandemics

Angelini, A. L. (1979). O papel da Sociedade Interamericana de Psicologia no desenvolvimento da psicologia na América Latina. Revista Interamericana de Psicologia/Interamerican Journal of Psychology, 13(1-2), 5-25.

Ardila, R. (1986). La psicología en América Latina. Pasado, presente, y futuro. Siglo XXI.

Baikie, K. A., \& Wilhelm, K. (2005). Emotional and physical health benefits of expressive writing. Advances in Psychiatric Treatment, 11(5), 338-346. https://doi.org/10.1192/apt.11.5.338

Bloj, A. (6 de abril, 2020). Coronavirúsico [Video]. Youtube. https://www.youtube.com/watch?v=70UKrH6cpbw

Brooks, S. K., Webster, R. K., Smith, L. E., Woodland, L., Wessely, S., Greenberg, N., Rubin, G. J. (2020). The psychological impact of quarantine and how to reduce it: rapid review of the evidence. The Lancet, 395, 912-920. https://doi.org/10.1016/S0140-6736(20)30460-8.

Calva, E. A., \& López, M. E. G. (2007). Criterios para la elaboración de las guías de intervención psicológica. Perinatología y Reproducción Humana, 21(1), 11-21.

Centers for Disease Control and Prevention (2020). Coronavirus (COVID-19). https://www.cdc.gov/coronavirus/2019-nCoV/index.html

Charney, D. S. (2004). Psychobiological mechanisms of resilience and vulnerability: implications for successful adaptation to extreme stress. American Journal of Psychiatry, 161(2), 195-216. https://doi.org/ 10.1176/appi.ajp.161.2.195

Chorpita, B.F., Albano, A. M., \& Barlow, D. H. (1996). Cognitive processing in children: Relation to anxiety and family influences. Journal of Clinical Child Psychology, 25(2), 170-176. doi: https://doi.org/10.1207/s15374424jccp2502_5

Coetzer, R. \& Bichard, H. (2020). The challenges and opportunities of delivering clinical neuropsychology services during the Covid-19 crisis of 2020. Cuadernos de Neuropsicología/Panamerican Journal of Neuropsychology, 14(1), 10-18. https://doi.org/10.7714/CNPS/14.1.204

Joint Task Force for the Development of Telepsychology Guidelines for Psychologists. (2013). Guidelines for the practice of telepsychology. Traducción al español. https://doi.org/10.23923/cop.telepsicologia.2017

Colegio Oficial de la Psicología de Madrid. (2016). Guía para la intervención psicológica con migrantes y refugiados. https://www.copmadrid.org/web/publicaciones/guia-para-la-intervencionpsicologica-con-inmigrantes-y-refugiados

Colegio Oficial de la Psicología de Madrid. (2020a). Recomendaciones dirigidas a la población para un afrontamiento eficaz ante el malestar psicológico generado por el coronavirus-Covid 19. 
https://www.copmadrid.org/web/files/comunicacion/Comunicado_recomendaci ones_para_la_poblacion.pdf

Colegio Oficial de la Psicología de Madrid. (2020b). Orientaciones para la gestión psicológica de la cuarentena por el coronavirus. https://www.copmadrid.org/web/files/comunicacion/Orientaciones para la gest ion_psicologica_de_la_cuarentena_por_el_Coronavirus.pdf

Colegio Oficial de la Psicología de Madrid. (2020c). Recomendaciones psicológicas para explicar a niños y niñas el brote de coronavirus-Covid 19. http://www.infocop.es/view_article.asp?id=8626\&cat=8

Consoli, A. J., Morgan Consoli, M. L., Klappenbach, H., Sheltzer, J., \& Romero, A. (2015). Psychology in Latin America: Legacies and contributions-Part 3. International Psychology Bulletin, 19(2), 34-40.

Echavarría, Ramírez, D., Díaz-Reyes, V., \& Narzisi, A. (2020). Trastorno del espectro autista: pautas para el manejo durante el periodo de aislamiento social por el coronavirus. Cuadernos de Neuropsicología/Panamerican Journal of Neuropsychology, 14(1), 10-18. https://doi.org/10.7714/CNPS/14.1.205

Fancourt, D., \& FinnWhat, S. (2019). What is the evidence on the role of the arts in improving health and well-being? A scoping review. Health Evidence Network synthesis report 67. World Health Organization (WHO).

Ferdman, B. M., \& VanOss Marin, B. (1999). Interamerican Society of Psychology: History and current status. En M. M. Alonso \& A. Eagly (Eds.), Psicologia en las Americas (pp. 353-359). Sociedad Interamericana de Psicología.

Fernández Millán, J. (2013). Gestión e intervención psicológica en emergencias y catástrofes. Pirámide.

Gallegos, M. (2012). Historia de la psicología interamericana: Sociedad Interamericana de Psicología (1951). Psychologia Latina, 3(1), 23-36.

Gallegos, M. (2012). El Primer Congreso Interamericano de Psicología (1953): su acontecer histórico. Revista Interamericana de Psicología/Interamerican Journal of Psychology, 46(1), 21-34.

Gallegos, M. (2013). Sixty years of the Interamerican Society of Psychology: origins and developments. International Journal of Psychology, 48(6), 1313-1320.

Gallegos, M., Salas, G., Ardila, R., Caycho-Rodríguez, T., y Burgos, C. (2018). Sociedad Interamericana de Psicología: historia y proyección estratégica. Revista Mexicana de Psicología, 35(1), 72-87.

Gallegos, M., Zalaquett, C., Luna, S. E., Mazo, R., Ortiz, B., Penagos-Corzo, J. C., Portillo, P., Torres-Fernández, I., Urzúa, A., Morgan-Consoli, M., Polanco, F., Florez, A. M. \& Lopes Miranda, R. (2020). Protocolo de Acción y Recomendaciones sobre Salud Mental para afrontar la Pandemia del Coronavirus. Responsabilidad, Empatía, Solidaridad y Tranquilidad. Recuperado de https://sipsych.org/wp-content/uploads/2020/03/SIP-2020-CORONAVIRUS18_03_2020-Español.pdf

Garrick, J. (2006). The humor of trauma survivors: Its application in a therapeutic milieu. Journal of Aggression, Maltreatment \& Trauma, 12(1-2), 169-182. https://doi.org/10.1300/J146v12n01_09

Gisbert, J. P., Alonso-Coello, P., \& Piqué, J. M. (2008). How can we find, design, evaluate and use clinical practice guidelines?. Gastroenterologia $y$ Hepatologia, 31(4), 239-257. doi: 10.1157/13117903

Guil-Bozal, R., González-Fernández S., Mestre J. M., Zayas, A., Guerrero-Rodríguez, C., Gil-Olarte, P. (2018). New forms of social interaction: Virtual communication and health. Duazary, 15(3), 263-272. http://dx.doi.org/10.21676/2389783X.2417 
Harapan, H., Itoh, N., Yufika, A., Winardi, W., Keam, S., Te, H., Megawati, D., Hayati, Z., Wagner, A. L., \& Mudatsir, M. (2020). Coronavirus disease 2019 (COVID19): A literature review. Journal of Infection and Public Health, 13(5), 667673. https://doi.org/10.1016/j.jiph.2020.03.019

Hernández, S. M., Amezcua, M., Gómez, J. L., \& Hueso, C. (2015). Cómo elaborar Guías de Recomendaciones de Autocuidado para Pacientes y Cuidadores (GRAPyC). Index de Enfermería, 24(4), 245-249.

Huarcaya-Victoria, J. (2020). Consideraciones sobre la salud mental en la pandemia de COVID-19. Revista Peruana de Medicina Experimental y Salud Pública, 37(2) https://doi.org/10.17843/rpmesp.2020.372.5419

Inter-Agency Standing Committee (2009). Guía del IASC sobre Salud Mental y Apoyo Psicosocial en Emergencias Humanitarias y Catástrofes. Disponible en: https://www.who.int/hac/techguidance/iascguidelinesspanish.pdf?ua=1

Inter-Agency Standing Committee. (2020). Briefing note on addressing mental health and psychosocial aspects of COVID-19 OutbreakVersion 1.1. https://interagencystandingcommittee.org/system/files/202003/MHPSS\%20COVID19\%20Briefing\%20Note\%202\%20March\%202020English.pdf

Limaye, R. J., Sauer, M., Ali, J., Bernstein, J., Wahl, B., Barnhill, A., \& Labrique, A. (2020). Building trust while influencing online COVID-19 content in the social media world. The Lancet: Digital Health. doi: https://doi.org/10.1016/S25897500(20)30084-4

Lippi, G., Henry, B., Bovo, C., \& Sanchis-Gomar, F. (2020). Health risk and potential remedies during prolonged lockdowns for coronavirus disease 2019 (COVID-19). Diagnosis, 7(2):85-90. https//doi.org/10.1515/dx-2020-0041

López-Cañón, L. M., \& Pérez-Acosta, A. M. (2020). Covid-19, automedicación y cuarentena. SIP Bulletin. Número Especial Covid-19 (en prensa). https://sipsych.org/publications/sip-bulletin/

Lorenzo Ruiz, A., \& Guerrero, A. (2017). Afectaciones psicológicas en personal de primera respuesta: ¿trastorno por estrés postraumático o estrés traumático secundario? Revista Puertorriqueña de Psicología, 28(2), 252-265.

Lorenzo Ruiz, A., Díaz Arcaño, K., \& Zaldívar Pérez, D. (2020). La psicología como ciencia en el afrontamiento a la COVID-19: apuntes generales. Anales de la Academia Ciencia de Cuaba, 10(2). http://revistaccuba.sld.cu/index.php/revacc/article/view/839/856

Martín, L., \& Muñoz, M. (2009). Primeros auxilios psicológicos. Síntesis.

Martínez, R., Pastor, L., Pau, L., Pereira, P. \& Vela, B. (2016). El maletín del psicólogo en centro y servicios de atención a personas con discapacidad intelectual. Plena Inclusión Madrid.

Molina, M. (12 de marzo, 2020). Hola! Soy el Coronavirus. Un cuento para explicarles a los chicos sobre el coronavirus [Video]. Youtube. https://www.youtube.com/watch?v=ah0U6xbm8po

National Institute for Health and Care Excellence (2017). Mental health of adults in contact with the criminal justice system https://www.nice.org.uk/guidance/ng66

Organización Panamericana de la Salud/Organización Mundial de la Salud (2020). Consideraciones psicosociales y de salud mental durante el brote de COVID-19. https://www.paho.org/es/documentos/consideraciones-psicosociales-saludmental-durante-brote-covid-19

Organización Mundial de la Salud (2020). Preguntas y respuestas sobre la enfermedad por

(COVID-19). 
https://www.who.int/es/emergencies/diseases/novel-coronavirus-2019/advicefor-public/q-a-coronaviruses

Pautassi, R. \& Bonino, P. (Eds.) (2020). Del "decí que no..." al "sabías que...?”: ciencia y políticas públicas para la prevención del consumo del alcohol en jóvenes. Ministerio de Salud de la Provincia de Córdoba.

Penagos-Corzo, J. C., Tolamatl, C. R., Espinosa, A., Lorenzo Ruiz, A., \& Pintado, S. (2019). Psychometric Properties of the PTGI and Resilience in Earthquake Survivors in Mexico. Journal of Loss and Trauma, 1-21. https://doi.org/10.1080/15325024.2019.1692512

Pérez-Acosta, A. M. (2020). Automedicación y COVID-19. Revista Rosarista Nova et Vetera, 6(58). $\quad$ https://www.urosario.edu.co/Revista-Nova-EtVetera/Omnia/Automedicacion-y-covid-19/

Pineda Roa, A. (2020). Lineamientos y acciones de salud sexual para afrontar la pandemia del Coronavirus [Manuscrito no publicado]. Grupo de Trabajo "Psicología y Diversidades Sexuales". Sociedad Interamericana de Psicología.

Quezada-Schol, V. (2020). Miedo y psicopatología la amenaza que oculta el Covid-19. Cuadernos de Neuropsicología/Panamerican Journal of Neuropsychology, 14(1), 19-23. https://doi.org/10.7714/CNPS/14.1.202

Rodríguez, J., Zacarelli, M., Pérez, R., \& OPS/OMS. (2006). Guía práctica de salud mental en situaciones de desastres. Organización Panamericana de la Salud. Organización Mundial de la https://www.paho.org/hq/dmdocuments/2008/GuiaPracticadeSaludMental.pdf

Rojas-Jara, C. (2019). Drogas, drogos y drogodependencias: reformulando el objeto, el sujeto y el tratamiento psicológico del consumo problemático de drogas. En C. Rojas-Jara (Ed), Drogas: sujeto, sociedad y cultura (pp.107-122). Nueva Mirada.

Rojas-Jara, C. (2020). Cuarentena, aislamiento forzado y uso de drogas. Cuadernos de Neuropsicología/Panamerican Journal of Neuropsychology, 14(1), 42-28. https://doi.org/10.7714/CNPS/14.1.203

Rosenberg, A. R. (2020). Cultivating deliberate resilience during the Coronavirus Disease 2019 pandemic. JAMA Pediatrics, E1-E2. doi: https://doi.org/10.1001/jamapediatrics.2020.1436

Roth, S., \& Cohen, L. J. (1986). Approach, avoidance, and coping with stress. American Psychologist, 41(7), 813-819. https://doi.org/10.1037/0003-066X.41.7.813

Santrock, J. W. (2019). Adolescence (17th ed.). McGraw-Hill.

Sedlmeier, P., Eberth, J., Schwarz, M., Zimmermann, D., Haarig, F., Jaeger, S., \& Kunze, S. (2012). The psychological effects of meditation: A meta-analysis. Psychological Bulletin, 138(6), 1139-1171. https://doi.org/10.1037/a002816

Sim, K., \& Chua, H.C. (2014). The psychological impact of SARS: a matter of heart and mind. Canadian Medical Association Journal, 170(5), 811-812

Snider, L. van Ommeren, M., \& Schafer, A. (2012). Primera ayuda psicológica: guía para trabajadores de campo. Organización Mundial de la Salud

Torales, J., O’Higgins, M., Castaldelli-Maia, J. M., \& Ventriglio, A. (2020). The outbreak of COVID-19 coronavirus and its impact on global mental health. International Journal of Social Psychiatry, 31, 1-4. https://doi.org/10.1177/0020764020915212

United Nations (2020). Policy Brief: The Impact of COVID-19 on Women. https://www.unwomen.org//media/headquarters/attachments/sections/library/publications/2020/policybrief-the-impact-of-covid-19-on-women-en.pdf?la=en\&vs=1406 
Urzúa, A., Vera-Villarroel, P., Caqueo-Urízar, A. \& Polanco-Carrasco, R, (2020). La psicología en la prevención y manejo del COVID-19. Aportes desde la evidencia inicial. Terapia Psicológica, 38(1), 103-118.

Vera-Villarroel, P. (2020). Covid-19: un análisis desde los procesos psicológicos básicos. Cuadernos de Neuropsicología/Panamerican Journal of Neuropsychology, 14(1), 10-18. https://doi.org/s10.7714/CNPS/14.1.201

Wipfli, B. M., Rethorst, C. D., \& Landers, D. M. (2008). The anxiolytic effects of exercise: A meta-analysis of randomized trials and dose-response analysis. Journal of Sport \& Exercise Psychology, 30, 392-410. https://doi.org/10.1123/jsep.30.4.392

World Health Organization. (2015). Humanitarian Intervention Guide (mhGAP-HIG): clinical management of mental, neurological and substance use conditions in humanitarian emergencies. https://iris.paho.org/handle/10665.2/28418

World Health Organization. (2020a). Mental Health Considerations during COVID-19 Outbreak. https://www.who.int/docs/default-source/coronaviruse/mental-healthconsiderations.pdf?sfvrsn=6d3578af 2

World Health Organization. (2020b). Preparedness, prevention and control of COVID19 in prisons and other places of detention. http://www.euro.who.int/data/assets/pdf_file/0019/434026/Preparednessprevention-and-control-of-COVID-19-in-prisons.pdf

Worden, J. W. (1997). El tratamiento del duelo. Asesoramiento psicológico y terapia. Paidós. 